\title{
Age, left atrial dimension and arterial stiffness after external cardioversion of atrial fibrillation. A vascular component in arrhythmia maintenance? Results from a preliminary study
}

\author{
Stefano Fumagalli • Debbie Gabbai - Besmir Nreu • Anna T. Roberts • \\ Serena Boni - Alice Ceccofiglio $\cdot$ Stefania Fracchia $\cdot$ Samuele Baldasseroni · \\ Francesca Tarantini $\cdot$ Niccolò Marchionni
}

Received: 6 September 2013 / Accepted: 13 November 2013/Published online: 24 November 2013

(C) The Author(s) 2013. This article is published with open access at Springerlink.com

\begin{abstract}
Background and Aims Atrial fibrillation (AF) is the most frequent arrhythmia in elderly patients. Aims of this study were to evaluate the predictors of arterial stiffness after external cardioversion (ECV) of $\mathrm{AF}$ and to establish whether a link exists between vascular properties and left atrial diameter (LAD).

Methods We studied 33 patients (age $73 \pm 12$ years). After $5 \mathrm{~h}$ from $\mathrm{ECV}$ of persistent $\mathrm{AF}$, an echocardiogram was recorded and arterial stiffness was evaluated with cardio-ankle vascular stiffness index (CAVI).

Results In multivariate analysis $(R=0.538, p=0.006)$, CAVI (mean $9.60 \pm 1.63$ ) increased with age $(p=0.018)$ and with an AF length $\leq 3$ months $(p=0.022)$. LAD was significantly related to CAVI $(p=0.007)$ even after adjustment for interventricular septum thickness $(p=$ $0.018)(R=0.574, p=0.002)$.

Conclusions In patients with AF, immediately after ECV, arterial stiffness is associated with age and AF length, and could represent an important factor for left atrium remodeling and, therefore, for AF maintenance.
\end{abstract}

Keywords Arterial stiffness - Atrial fibrillation · CAVI · Elderly · External cardioversion · Left atrium diameter

S. Fumagalli $(\bowtie) \cdot$ D. Gabbai · B. Nreu •

A. T. Roberts $\cdot$ S. Boni $\cdot$ A. Ceccofiglio $\cdot$ S. Fracchia $\cdot$

S. Baldasseroni · F. Tarantini · N. Marchionni Intensive Care Unit and Geriatric Arrhythmology Unit, Division of Geriatric Cardiology and Medicine, and Research Unit of Medicine of Aging, Department of Experimental and Clinical Medicine, University of Florence and AOU Careggi, Viale Pieraccini, 6, 50139 Florence, Italy e-mail: fumadue@tin.it

\section{Introduction}

Atrial fibrillation (AF) is the most common sustained arrhythmia in the elderly [1]; its prevalence is justified by cardiovascular aging and the age-related increase of comorbidity [1-3]. Arterial stiffness (AS) also increases with age and predicts coronary heart disease, stroke and mortality [4]. The endpoint of this study was to define the clinical determinants of AS in subjects with persistent AF immediately after external cardioversion (ECV). Furthermore, we assessed the existence of a link between left atrium (LA) diameter (LAD) and AS.

\section{Methods}

Patients and procedures

We enrolled all patients admitted to a day-hospital for ECV of persistent AF between January and June 2013; the only exclusion criterion was non-restoration of SR. The protocol was approved by an institutional committee and conforms to the 1975 Declaration of Helsinki ethical guidelines; patients gave their consent to participate. ECV was performed using a biphasic defibrillator, after a 4-week period of effective oral anticoagulation [1]. An echocardiogram was digitally recorded $5 \mathrm{~h}$ after the procedure.

Measure of AS

The cardio-ankle vascular index (CAVI) [5], a measure of AS, independent from instantaneous systolic and pulse pressure, was determined using VaSera VS-1500N (Fukuda Denshi, Japan) after 20 min of rest in supine position. During the continuous recording of EKG and heart sounds, 
right and left upper and lower extremity arterial pressure was obtained with the oscillometric method. Pulse wave velocity (PWV) was calculated dividing the distance from the aortic valve to the ankle artery (an indirect measure derived from the height of the patient) by the sum of two time intervals [(1) aortic valve closing sound-notch of the brachial pulse wave; (2) rise of the brachial pulse waverise of the ankle pulse wave] [5]. CAVI was then computed using the following equation:

$\mathrm{CAVI}=a \times\left[(2 \rho / \Delta P) \times \ln \left(P_{\mathrm{s}} / P_{\mathrm{d}}\right) \times \mathrm{PWV}^{2}\right]+b$

where $P_{\mathrm{s}} / P_{\mathrm{d}}$ are systolic/diastolic pressures, $\Delta P$ is " $P_{\mathrm{s}}-$ $P_{\mathrm{d}}$ ", $\rho$ is blood density, and $a / b$ are constants [5]. CAVI was measured after SR restoration to avoid bias due to different RR intervals [5].

\section{Statistical analysis}

IBM SPSS for Windows (ver. 20) was used for statistical analysis. Continuous variables were expressed as mean $\pm \mathrm{SD}$, categorical variables as numbers with percentages. Student's $t$ test and analysis of variance were used to compare continuous variables. The association between categorical variables was evaluated with $\chi^{2}$ test. Linear regression analysis models were used to define univariate and multivariate predictors of CAVI and LAD. A $p$ value $<0.05$ was considered statistically significant.

\section{Results}

\section{Patients' description}

Thirty-three patients underwent ECV (Table 1). Median AF length was 3 months (range 1-18). Right arm arterial pressure and right ankle-brachial index were $140 \pm 16$ / $85 \pm 11 \mathrm{mmHg}$ and $1.10 \pm 0.15$, respectively, with no difference between right- and left-side measurements. Thromboembolic risk $\left(\mathrm{CHADS}_{2}\right.$ score) was moderate in $72.7 \%$ and high in $18.3 \%$ of cases. Beta-blockers, ACEinhibitors or angiotensin receptor blocking agents were the most frequently used cardiovascular drugs (Table 1). There was no difference between right and left CAVI $(9.60 \pm 1.63$ and $9.56 \pm 1.64$, respectively; $p=0.78)$. Therefore, only the results related to right CAVI will be presented.

\section{CAVI determinants}

In univariate analysis, CAVI was significantly associated with age $(R=0.390, \quad p=0.025), \quad$ BMI $(R=0.364$, $p=0.037$ ), the presence of mitral regurgitation (yes: $10.3 \pm 1.4$ vs. no: $8.9 \pm 1.6, p=0.012)$ and an $\mathrm{AF}$
Table 1 Patients' clinical characteristics

\begin{tabular}{|c|c|c|}
\hline \multicolumn{2}{|l|}{$(n=33)$} & \multirow{2}{*}{$\frac{\text { Range }}{35-90}$} \\
\hline Age (years) & $73 \pm 12$ & \\
\hline $\operatorname{Men}(n, \%)$ & $28(84.8)$ & \\
\hline Height $(\mathrm{cm})$ & $174 \pm 9$ & $150-192$ \\
\hline Weight (Kg) & $82 \pm 15$ & $56-138$ \\
\hline BMI $\left(\mathrm{Kg} / \mathrm{m}^{2}\right)$ & $27.0 \pm 5.0$ & $19.8-48.9$ \\
\hline Present smokers $(n, \%)$ & $7(21.2)$ & \\
\hline $\mathrm{CAD}(n, \%)$ & $6(18.2)$ & \\
\hline $\operatorname{CHF}(n, \%)$ & $11(33.3)$ & \\
\hline $\operatorname{CKD}(n, \%)$ & $5(15.2)$ & \\
\hline $\operatorname{CVD}(n, \%)$ & $2(6.1)$ & \\
\hline Diabetes $(n, \%)$ & $4(12.1)$ & \\
\hline Dyslipidemia $(n, \%)$ & $12(36.4)$ & \\
\hline Hypertension $(n, \%)$ & $22(66.7)$ & \\
\hline LAF/brady-tachycardia syndrome $(n, \%)$ & $6(18.2)$ & \\
\hline \multicolumn{3}{|l|}{ Valvular disease $(n, \%)$} \\
\hline Aortic regurgitation & $7(21.2)$ & \\
\hline Aortic stenosis/prosthesis & $4(12.1)$ & \\
\hline Mitral regurgitation & $17(51.5)$ & \\
\hline $\mathrm{CHADS}_{2}$ (score) & $1.8 \pm 1.3$ & $0-5$ \\
\hline Pulse Pressure (mmHg) & $57 \pm 13$ & $34-95$ \\
\hline $\mathrm{LAD}(\mathrm{mm})$ & $50 \pm 7$ & $35-63$ \\
\hline Interventricular septum (mm) & $10 \pm 1.5$ & $7-12$ \\
\hline LVEDD (mm) & $56 \pm 9$ & $43-86$ \\
\hline LVESD (mm) & $39 \pm 10$ & $24-71$ \\
\hline LVEF (\%) & $56 \pm 14$ & $20-79$ \\
\hline Hemoglobin (g/dL) & $14.0 \pm 1.7$ & $11.0-17.3$ \\
\hline Creatinine (mg/dL) & $0.9 \pm 0.3$ & $0.4-1.6$ \\
\hline $\operatorname{ACE}-\mathrm{I} / \mathrm{ARB}(n, \%)$ & $27(81.8)$ & \\
\hline$\beta$-blockers $(n, \%)$ & $25(75.8)$ & \\
\hline Ca-antagonists $(n, \%)$ & $5(15.2)$ & \\
\hline Digoxin $(n, \%)$ & $15(45.5)$ & \\
\hline Statins $(n, \%)$ & $9(27.3)$ & \\
\hline
\end{tabular}

$B M I$ body mass index, $C A D$ coronary artery disease, $C H F$ chronic heart failure, $C K D$ chronic kidney disease, $C V D$ cerebro-vascular disease, Interventricular septum Interventricular septum thickness, $L A F$ lone atrial fibrillation, Brady-Tachycardia Syndrome bradycardia-tachycardia syndrome, Prosthesis biological or mechanical aortic prosthetic valve, $L A D$ left atrium end-systolic diameter, $L V E D D /$ $L V E S D$ left ventricular end-systolic/end-diastolic diameter, $L V E F$ left ventricular ejection fraction, $A R B$ angiotensin receptor blocking agents, $\mathrm{Ca}$-antagonists dihydropyridine $\mathrm{Ca}$-antagonists

length $\leq 3$ months (yes: $10.1 \pm 1.5$ vs. no: $8.9 \pm 1.5$, $p=0.031$ ). CAVI was not affected by gender or current smoking status, nor did we find any link with arterial hypertension, CHF, chronic renal failure, coronary artery and cerebrovascular disease, diabetes, dyslipidemia or other causes of AF. Heart rate, systolic, diastolic and pulse pressure, hemoglobin concentration and drug therapy did not correlate with CAVI. Interventricular septum thickness, 
Fig. 1 Association between left atrium diameter and CardioAnkle Vascular Index (left) and interventricular septum thickness (right) in patients with persistent atrial fibrillation evaluated after successful external cardioversion (univariate regression analysis model)

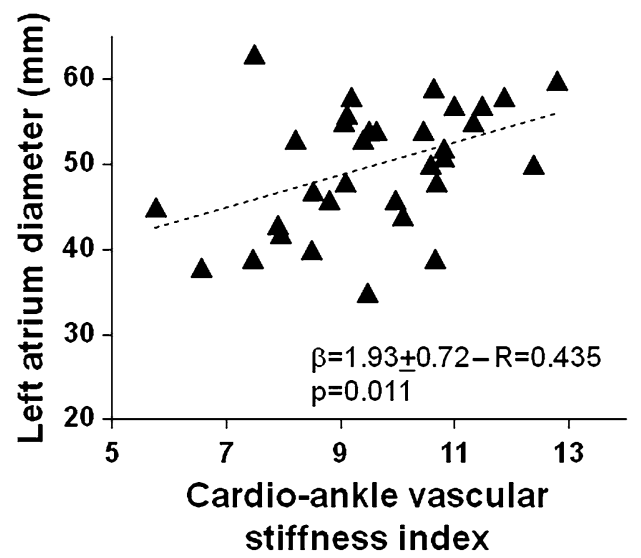

left ventricular (LV) diameters, and LVEF were not associated with CAVI, but LAD was $(R=0.435, p=0.011)$. A multivariate regression analysis model $(R=0.538$, $p=0.006)$ showed that age $(\beta=0.05 \pm 0.02,95 \%$ $\mathrm{CI}=0.01-0.09, p=0.018)$ and an AF length $\leq 3$ months $(\beta=1.20 \pm 0.50,95 \% \mathrm{CI}=0.18-2.22, p=0.018)$ were significantly related to CAVI, while BMI lost its statistical significance $(p=0.11)$.

\section{LAD determinants}

LAD was not associated with age, gender, BMI, comorbid conditions, mitral regurgitation, $\mathrm{AF}$ length, drugs, arterial pressure, LV diameters, LVEF or hemoglobin concentration. Apart from CAVI, only interventricular septum thickness showed a direct association with atrial dimensions $(R=0.378$, $p=0.030$ ). A multivariate model (overall $R=0.574$, $p=0.002$ ) demonstrated that both interventricular septum thickness $\quad(\beta=1.82 \pm 0.73, \quad 95 \% \quad \mathrm{CI}=0.34-3.30$, $p=0.018) \quad$ and $\quad$ CAVI $\quad(\beta=1.92 \pm 0.66, \quad 95 \%$ $\mathrm{CI}=0.56-3.27, p=0.007$ ) were predictors of LAD (Fig. 1).

\section{Discussion}

The results of this preliminary study confirm the existence of a direct relationship between CAVI and age, also in patients treated with ECV for persistent AF. Interestingly, a shorter length of AF seems to be associated with higher CAVI values. We also showed that $\mathrm{AS}$, together with interventricular septum thickness, is a significant determinant of LAD.

The inverse correlation between CAVI and duration of AF, observed in the first few months after arrhythmia development, might be attributed to the increase in afterload due to the early effects on cardiac performance associated with the loss of atrial systole [6].

Few data exist aimed at clarifying the interaction among $\mathrm{AF}, \mathrm{LAD}$ and $\mathrm{AS}$, and results are sometimes conflicting. In a previous study, Reiffel [7] found that AS and LV hypertrophy did not predict the risk of AF in hypertensive patients and concluded that the arrhythmia is generated by complex mechanisms induced by atrial hypertension. On the contrary, Lantelme and colleagues [8] showed that in hypertensive patients LAD correlated with PWV or pulse pressure, suggesting that AF could be a link between AS and stroke. Finally, patients with obstructive sleep apnea showed a direct correlation between LAD and AS [9]. LA and LV act in synergy; atrial volume has been proven to express the severity of ventricular diastolic dysfunction in patients without atrial arrhythmias or valvular heart disease [10]. However, the association of LAD with diastolic dysfunction cannot fully explain our results. In fact, CAVI remained independently correlated with atrial dimensions even after adjustment for interventricular septum thickness, a parameter linked with altered LV relaxation [11]. A possible explanation of the link between AS and LAD we found in this study could be represented by mitral regurgitation, present in $51.5 \%$ of our patients and associated with higher CAVI values. In addition, AS seems to promote inflammation, vascular smooth muscle cell contraction, and stretch-induced release of metalloproteinase-12 in atrial tissues [12]. To further stress the importance of nonmechanical factors in the complex network linking LA, LV and arterial properties, a recent review suggests that comorbidities can produce coronary micro-vascular dysfunction, mediated by inflammation, ultimately leading to myocardial stiffness progression [13]. All these data support the existence of a connection between AS and AF.

\section{Study limitations}

The study is preliminary and the sample is small, with only 5 women. The echocardiogram was recorded $5 \mathrm{~h}$ after effective ECV; yet, current evidence seems to suggest that such a short period of SR is unable to revert the electrical and structural changes which characterize atrial remodeling [14]. Finally, our results might have been different had we 
performed pharmacological cardioversion; however, ECV is the treatment of choice for AF lasting $>48 \mathrm{~h}$ [1].

In conclusion, the results of our preliminary study support the hypothesis that age-related AS is independently associated with atrial size in patients with persistent AF. Therefore, the concept that "atrial fibrillation begets atrial fibrillation" [15] might rely also on a "vascular" component. Further studies are needed to confirm present findings and to clarify if AS may exert a role in arrhythmia recurrence.

Conflict of interest On behalf of all authors, the corresponding author states that there is no conflict of interest.

Open Access This article is distributed under the terms of the Creative Commons Attribution License which permits any use, distribution, and reproduction in any medium, provided the original author(s) and the source are credited.

\section{References}

1. Camm AJ, Kirchhof P, Lip GY, Schotten U, Savelieva I, Ernst S et al (2010) Guidelines for the management of atrial fibrillation: the task force for the management of atrial fibrillation of the European Society of Cardiology (ESC). Eur Heart J 31:2369-2429

2. Fumagalli S, Tarantini F, Guarducci L, Pozzi C, Pepe G, Boncinelli L et al (2010) Atrial fibrillation is a possible marker of frailty in hospitalized patients: results of the GIFA Study. Aging Clin Exp Res 22:129-133

3. Testa G, Cacciatore F, Della-Morte D, Galizia G, Mazzella F, Gargiulo $G$ et al (2012) Role of permanent atrial fibrillation (AF) on long-term mortality in community-dwelling elderly people with and without chronic heart failure (CHF). Arch Gerontol Geriatr 55:91-95

4. Cavalcante JL, Lima JA, Redheuil A, Al Mallah MH (2011) Aortic stiffness: current understanding and future directions. J Am Coll Cardiol 57:1511-1522
5. Shirai K, Hiruta N, Song M, Kurosu T, Suzuki J, Tomaru T et al (2011) Cardio-ankle vascular index (CAVI) as a novel indicator of arterial stiffness: theory, evidence and perspectives. J Atheroscler Thromb 18:924-938

6. Zhang C, Ohira M, Iizuka T, Mikamo H, Nakagami T, Suzuki M et al (2013) Cardio-ankle vascular index relates to left ventricular ejection fraction in patients with heart failure. A retrospective study. Int Heart J 54:216-221

7. Reiffel JA (2004) Is arterial stiffness a contributing factor to atrial fibrillation in patients with hypertension? A preliminary investigation. Am J Hypertens 17:213-216

8. Lantelme P, Laurent S, Besnard C, Bricca G, Vincent M, Legedz L et al (2008) Arterial stiffness is associated with left atrial size in hypertensive patients. Arch Cardiovasc Dis 101:35-40

9. Drager LF, Bortolotto LA, Pedrosa RP, Krieger EM, LorenziFilho G (2010) Left atrial diameter is independently associated with arterial stiffness in patients with obstructive sleep apnea: potential implications for atrial fibrillation. Int $\mathrm{J}$ Cardiol 144:257-259

10. Tsang TS, Barnes ME, Gersh BJ, Bailey KR, Seward JB (2002) Left atrial volume as a morphophysiologic expression of left ventricular diastolic dysfunction and relation to cardiovascular risk burden. Am J Cardiol 90:1284-1289

11. De Marchi SF, Allemann Y, Seiler C (2000) Relaxation in hypertrophic cardiomyopathy and hypertensive heart disease: relations between hypertrophy and diastolic function. Heart 83:678-684

12. Luft FC (2012) Molecular mechanisms of arterial stiffness: new insights. J Am Soc Hypertens 6:436-438

13. Paulus WJ, Tschope C (2013) A novel paradigm for heart failure with preserved ejection fraction: comorbidities drive myocardial dysfunction and remodeling through coronary microvascular endothelial inflammation. J Am Coll Cardiol 62:263-271

14. Kumar S, Teh AW, Medi C, Kistler PM, Morton JB, Kalman JM (2012) Atrial remodeling in varying clinical substrates within beating human hearts: relevance to atrial fibrillation. Prog Biophys Mol Biol 110:278-294

15. Wijffels MC, Kirchhof CJ, Dorland R, Allessie MA (1995) Atrial fibrillation begets atrial fibrillation. A study in awake chronically instrumented goats. Circulation 92:1954-1968 